MATHEMATICS OF COMPUTATION

Volume 75, Number 255, July 2006, Pages 1155-1174

S 0025-5718(06)01835-7

Article electronically published on February 22, 2006

\title{
A VARIANT OF THE LEVEL SET METHOD AND APPLICATIONS TO IMAGE SEGMENTATION
}

\author{
JOHAN LIE, MARIUS LYSAKER, AND XUE-CHENG TAI
}

\begin{abstract}
In this paper we propose a variant of the level set formulation for identifying curves separating regions into different phases. In classical level set approaches, the sign of $n$ level set functions are utilized to identify up to $2^{n}$ phases. The novelty in our approach is to introduce a piecewise constant level set function and use each constant value to represent a unique phase. If $2^{n}$ phases should be identified, the level set function must approach $2^{n}$ predetermined constants. We just need one level set function to represent $2^{n}$ unique phases, and this gains in storage capacity. Further, the reinitializing procedure requested in classical level set methods is superfluous using our approach. The minimization functional for our approach is locally convex and differentiable and thus avoids some of the problems with the nondifferentiability of the Delta and Heaviside functions. Numerical examples are given, and we also compare our method with related approaches.
\end{abstract}

\section{INTRODUCTION TO RELATED LEVEL SET METHODS}

A function $u: \Omega \mapsto \mathbb{R}$ defined on an open and bounded domain $\Omega \in \mathbb{R}^{m}$ may have different properties in distinct regions of $\Omega$. In many applications, one needs to separate $\Omega$ into a union of subdomains, i.e., $\Omega=\bigcup_{i=1}^{n} \Omega_{i}$, so that distinctive properties of $u$ can be linked to different subdomains and incorporated into a modeling process. Several approaches have been used to find a propitious partition of $\Omega$, and some of them are described briefly below. For the purpose of illustration, only the two-dimensional case is considered here.

One approach to finding a partition of $\Omega$ is to use an active contour. The basic idea is to let a closed curve $C$ separate $\Omega$ into two disjunctive subdomains [12]. Denote the region inside $C$ by $\Omega_{1}$, and the region outside $C$ by $\Omega_{2}$. The curve evolves, subject to constraints from $u$ together with some smoothness requirements of $C$. Traditionally, the curve $C$ is parameterized as $\mathbf{x}=\mathbf{x}(s), s \in[0,1]$. A convenient way to approximate an explicit representation of $C$, is to divide $s$ into a finite set of points $0=s_{0}<\cdots<s_{i-1}<s_{i}<s_{i+1} \cdots<s_{n}=1$, where the subintervals $\left[s_{i}, s_{i+1}\right]$ can vary in size, and then update the particle $\mathbf{x}$ in an iterative way. For some applications, it might be difficult to handle geometrical and topological changes of the curve using this kind of explicit approach. Numerical instability could also be a problem, since the points on the curve may get clustered.

Received by the editor March 12, 2004 and, in revised form, December 9, 2004.

2000 Mathematics Subject Classification. Primary 35G25, 65K10.

Key words and phrases. Level set, energy minimization, partial differential equations, segmentation.

This work was supported by the Norwegian Research Council. 


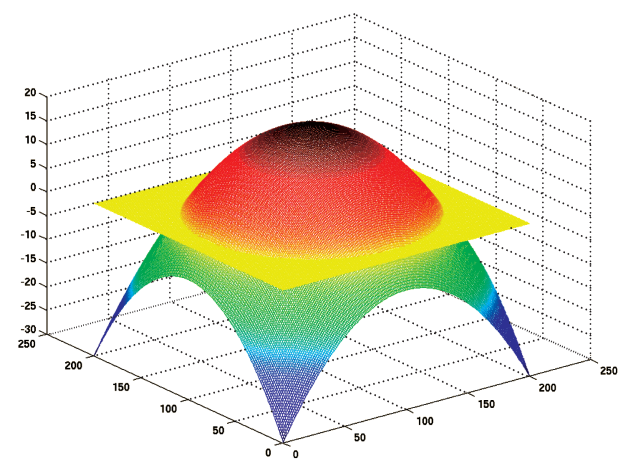

(a) The level set function $\phi$.

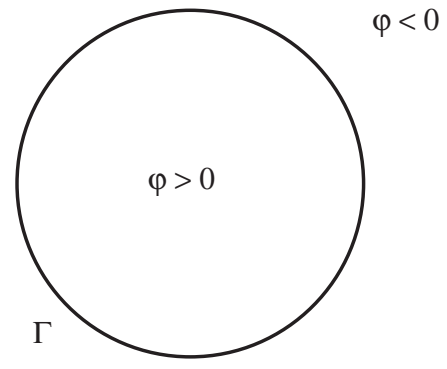

(b) The interface $\Gamma$.

Figure 1. Plot of the higher dimension function $\phi(\mathbf{x})$ and the interface $\Gamma=\{\mathbf{x} \in \Omega, \phi(\mathbf{x})=0\}$.

A successful way for representing an evolving curve is the level set method invented by Osher and Sethian in [18. The level set framework is now used on a broad spectrum of problems, including image analysis, reservoir simulations, inverse problems, computer vision and optimal shape design [1, 6, 5, 2, 24, 26]. With the level set representation, topological changes such as merging and breaking of curves can be handled in an efficient way. The curve that divides $\Omega$ into two subdomains is represented implicitly as the zero level set curve $\Gamma=\{\mathbf{x} \in \Omega, \phi(\mathbf{x})=0\}$ of a higher dimension level set function $\phi$. Rather than evolving the curve itself, the level set approach builds $\Gamma$ into the function $\phi$. Normally, $\phi$ is initialized as the signed distance function:

$$
\phi(\mathbf{x})=\left\{\begin{aligned}
\operatorname{distance}(\mathbf{x}, \Gamma), & \mathbf{x} \in \text { interior of } \Gamma \\
-\operatorname{distance}(\mathbf{x}, \Gamma), & \mathbf{x} \in \text { exterior of } \Gamma
\end{aligned}\right.
$$

In Figure 1(a) a simple level set function $\phi$ is illustrated, and its associated interface $\Gamma$ is given in Figure 1(b). $\Gamma$ divides $\Omega$ into two separate sub-domains. By moving $\phi$ up or down at different locations, the interface evolves. To divide $\Omega$ into more than two domains, multiple level set functions are needed [27, 19, 25. One of the concerns of multiple level set methods is to avoid vacuum and overlap between the different phases [27]. In [25], the following approach was used to create multiple phases without vacuum and overlaps. Assume we introduce two closed curves, $\Gamma_{1}$ and $\Gamma_{2}$, where each curve $\Gamma_{i}$ is associated with a level set function $\phi_{i}, i=1,2$. The domain $\Omega$ can now be divided into 4 parts:

$$
\begin{aligned}
& \Omega_{1}=\left\{\mathbf{x} \in \Omega, \quad \phi_{1}>0, \quad \phi_{2}>0\right\}, \quad \Omega_{2}=\left\{\mathbf{x} \in \Omega, \quad \phi_{1}>0, \quad \phi_{2}<0\right\}, \\
& \Omega_{3}=\left\{\mathbf{x} \in \Omega, \quad \phi_{1}<0, \quad \phi_{2}>0\right\}, \quad \Omega_{4}=\left\{\mathbf{x} \in \Omega, \quad \phi_{1}<0, \quad \phi_{2}<0\right\} \text {. }
\end{aligned}
$$

By further generalization, $n$ level set functions give the possibility of $2^{n}$ regions $[27$, 6]. These regions will be disjunct (no overlap) and their union is

$$
\Omega=\bigcup_{i} \Omega_{i} \cup \Gamma_{i}
$$


In this work, we propose an alternative approach. Assume that we need to find $n$ regions $\left\{\Omega_{i}\right\}_{i=1}^{n}$ which form a portion of $\Omega$. Some of the regions can be empty. This means that we can over-estimate the total number of phases for practical applications, and superfluous phases will disappear at convergence. In order to find the regions, we just need to form a piecewise constant function which takes values

$$
\phi=i \text { in } \Omega_{i}, \quad i=1,2, \ldots, n .
$$

The discontinuities of $\phi$ give us the curves separating the regions. For some applications, we could formulate the identification of the curves as a minimization problem with respect to $\phi$ under some constraints. This level set formulation can be applied in different applications where the classical level set methods are used today. In this work, we restrict ourselves to one special case: image segmentation. For such an application, we formulate the segmentation problem as a minimization problem with respect to $\phi$. See also our related work [14, where we use a set of functions $\left\{\phi_{i}\right\}_{i=1}^{n}$ to represent the discontinuities, and where each function only takes two values, i.e., $\phi_{i}= \pm 1$.

The idea of using one level set function to represent several phases is however not completely new. In island dynamics models for epitaxial growth of thin films, a large number of individual interfaces have to be identified. In [16, 7] they use one level set function to track islands on different monolayers, i.e., they let $\phi=0$ represent the island boundaries of the first monolayer, $\phi=1$ represent the island boundaries of the second monolayer and so on. By using just one level set function rather than one per layer, their algorithm is kept simple and memory costs are kept low. We will use a similar idea to track different segments in an image. However, to get this terrace level set formulation to fit into a segmentation framework, some innovative work has been done in this paper. The segmentation problem is formulated as a minimization problem with a smooth cost functional under a constraint. Our constraint is analogous to the potential used in phase-field methods; see [20]. Further, we would like to mention that the idea for the piecewise constant level set method has also been used for image segmentation in 22, 10. Another related work is the so-called MBO scheme for diffusion generated motions 15. Integer values $1,2, \ldots$ are used to represent the phases, and a projection forces the values to be integers. This scheme has recently been used for image segmentation in [8]. There are mainly two differences between our method and the method of [15, 8]. First, we use the augmented Lagrangian to deal with the constraint, while they use a projection to deal with the same constraint. Second, we use total variation norm for the regularization and thus preserve jumps in the functions. The schemes of 15, 8, are essentially using the $H^{1}$ norm for the phase function, and the scheme only has meaning when the regularization parameter goes to zero. In addition, discontinuities will be smeared out due to this.

Let us conclude this first section with the outline of this paper. In Section 2 we introduce a functional motivated from an optimal approximation problem. For the approximation problem we will use the partition described in (1.3) to express a general function as a piecewise constant function. In Section 3 the augmented Lagrangian method is introduced as a tool to solve the optimal approximation problem. In that section we also describe our algorithm, together with some other implementation details. The novelty in our approach, compared with classical level set methods, is reported in Section 4. Finally, Section 5 is devoted to numerical examples. 


\section{Representing PIECEWISE CONSTANT FUNCTIONS}

We use image segmentation as an example to show that our method can be utilized to identify curves separating regions. Let $u_{0}$ be a function defined in a domain $\Omega$, where $u_{0}$ reflects the intensity values for a given gray scale image, possibly imposed with noise. It is assumed that the intensity value of the true image is slowly varying inside each phase, but has large jumps between the phases. In order to find the discontinuities, we try to find a piecewise constant function approximating $u_{0}$. The discontinuities of the piecewise constant function will give us the boundary between the different phases.

In order to identify a piecewise constant function, we need to find the values for the constants and the location of the discontinuities. To find the locations of the discontinuities, the curve that separates the regions needs to be determined. Mumford and Shah [17] proposed to solve the "minimal" partition problem to approximate a function $u_{0}$ by a piecewise constant function $u$,

$$
\inf _{u, \Gamma}\left\{F^{M S}(u, \Gamma)=\sum_{i} \int_{\Omega_{i}}\left|c_{i}-u_{0}\right|^{2} d x+\nu|\Gamma|\right\},
$$

where $\Omega=\bigcup_{i} \Omega_{i} \cup \Gamma$. They tried to find a decomposition $\Omega_{i}$ of $\Omega$, where $u=c_{i}$ (constant) inside each component $\Omega_{i}$. The nonnegative parameter $\nu$ controls the length of the curve $\Gamma$. For a fixed $\Gamma$, we see that (2.1) is minimized when $c_{i}=$ mean $\left(u_{0}\right)$ in $\Omega_{i}$. A challenge when solving (2.1) is to find a unique representation of the parameterized curve $\Gamma$. To accommodate this problem, Chan and Vese [3] reformulated and solved (2.1) using a level set approach

$$
\begin{aligned}
\inf _{\mathbf{c}, \phi}\left\{F\left(c_{1}, c_{2}, \phi\right)=\right. & \int_{\Omega}\left|c_{1}-u_{0}\right|^{2} H(\phi) d x \\
& \left.+\int_{\Omega}\left|c_{2}-u_{0}\right|^{2}(1-H(\phi)) d x+\nu \int_{\Omega}|\nabla H(\phi)| d x\right\},
\end{aligned}
$$

where the Heaviside function $H(\phi)$ equals 1 if $\phi \geq 0$ and equals 0 if $\phi<0$. The regularization term $\int_{\Omega}|\nabla H(\phi)| d x$ measures the length of the interface and favors smooth curves 9 . Minimizing $F\left(c_{1}, c_{2}, \phi\right)$ with respect to $c_{1}$ and $c_{2}$, we obtain

$$
c_{1}=\operatorname{mean}\left(u_{0}\right) \text { if } \phi>0 \quad \text { and } \quad c_{2}=\operatorname{mean}\left(u_{0}\right) \text { if } \phi<0 .
$$

The active contour model without edges (2.2) can only divide $\Omega$ into two distinct regions. Later, Chan and Vese generalized this 2-phase active contour model to a multiphase level set framework [6, 25]. By using a multiphase level set representation, it is possible to solve the "minimal" partition problem (2.1) for an arbitrary number of partitions. Below we show the energy functional for a 4-phase partition, 
involving 2 level set functions:

$$
\begin{aligned}
F\left(\mathbf{c}, \phi_{1}, \phi_{2}\right)= & \int_{\Omega}\left|c_{1}-u_{0}\right|^{2} H\left(\phi_{1}\right) H\left(\phi_{2}\right) d x \\
& +\int_{\Omega}\left|c_{2}-u_{0}\right|^{2} H\left(\phi_{1}\right)\left(1-H\left(\phi_{2}\right)\right) d x \\
& +\int_{\Omega}\left|c_{3}-u_{0}\right|^{2}\left(1-H\left(\phi_{1}\right)\right) H\left(\phi_{2}\right) d x \\
& +\int_{\Omega}\left|c_{4}-u_{0}\right|^{2}\left(1-H\left(\phi_{1}\right)\right)\left(1-H\left(\phi_{2}\right)\right) d x \\
& +\nu \int_{\Omega}\left|\nabla H\left(\phi_{1}\right)\right| d x+\nu \int_{\Omega}\left|\nabla H\left(\phi_{2}\right)\right| d x .
\end{aligned}
$$

Once the constant values $\left\{c_{i}\right\}_{i=1}^{4}$ and the level set functions $\left\{\phi_{j}\right\}_{j=1}^{2}$ are identified, the piecewise constant function $u$ can be written as

$$
\begin{aligned}
u= & c_{1} H\left(\phi_{1}\right) H\left(\phi_{2}\right)+c_{2} H\left(\phi_{1}\right)\left(1-H\left(\phi_{2}\right)\right) \\
& +c_{3}\left(1-H\left(\phi_{1}\right)\right) H\left(\phi_{2}\right)+c_{4}\left(1-H\left(\phi_{1}\right)\right)\left(1-H\left(\phi_{2}\right)\right) .
\end{aligned}
$$

Given $u$ of this form, an alternative formulation of (2.3) was suggested in 5, 11. Here the authors tried to minimize the following energy functional directly with $u$ given as in (2.4):

$$
F\left(\mathbf{c}, \phi_{1}, \phi_{2}\right)=\int_{\Omega}\left|u-u_{0}\right|^{2} d x+\nu \int_{\Omega}\left|\nabla \phi_{1}\right| d x+\nu \int_{\Omega}\left|\nabla \phi_{2}\right| d x .
$$

The above functional is essentially an application of the functional given in [5] for the image segmentation problem. Omitting the regularization term, functional (2.3) equals functional (2.5). However, the gradients are not equal if we regularize the Heaviside function $H(\phi)$, and thus produce different numerical schemes. Numerical experiments in [11] indicate a somewhat better performance using the gradient associated with functional (2.5). Using the approaches above, two level set functions must be utilized to find four disjunct regions covering $\Omega$. Correspondingly, we need to identify $n$ functions and $2^{n}$ constants in order to identify $2^{n}$ phases.

Using our new approach as shown in (1.3), we just need one function to identify all the phases. Associated with $\phi$, we define a set of basis functions $\psi_{i}$ as

$$
\psi_{i}=\frac{1}{\alpha_{i}} \prod_{\substack{j=1 \\ j \neq i}}^{n}(\phi-j) \quad \text { and } \quad \alpha_{i}=\prod_{\substack{k=1 \\ k \neq i}}^{n}(i-k) .
$$

It is clear that a function $u$ given by

$$
u=\sum_{i=1}^{n} c_{i} \psi_{i}
$$

is a piecewise constant function and $u=c_{i}$ in $\Omega_{i}$ if $\phi$ is as given in (1.3). The sum in $u$ involves basis functions of polynomial functions of order $n-1$ in $\phi$ and the unknown coefficients $c_{i}, i=1,2, \ldots, n$. Each $\psi_{i}$ is expressed as a product of linear factors of the form $(\phi-j)$, with the $i$ th factor omitted. Thereupon $\psi_{i}(\mathbf{x})=1$ for 
$\mathbf{x} \in \Omega_{i}$, and $\psi_{i}(\mathbf{x})=0$ elsewhere as long as (1.3) holds. The basis functions can further be utilized to express arc length of $\partial \Omega_{i}$ and the area $\Omega_{i}$ of each phase, i.e.,

$$
\left|\partial \Omega_{i}\right|=\int_{\Omega}\left|\nabla \psi_{i}\right| d x \text { and }\left|\Omega_{i}\right|=\int_{\Omega} \psi_{i} d x .
$$

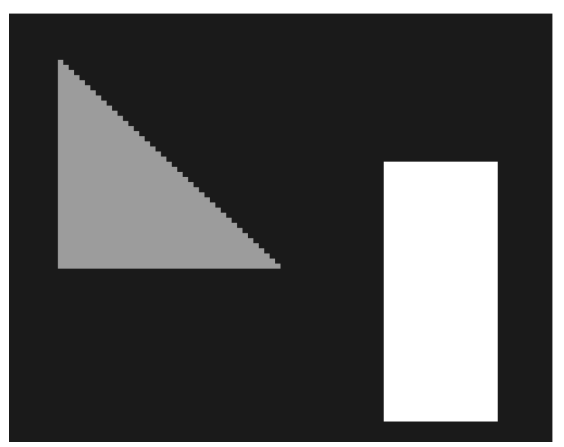

(a) Image $u$ with 3 objects.

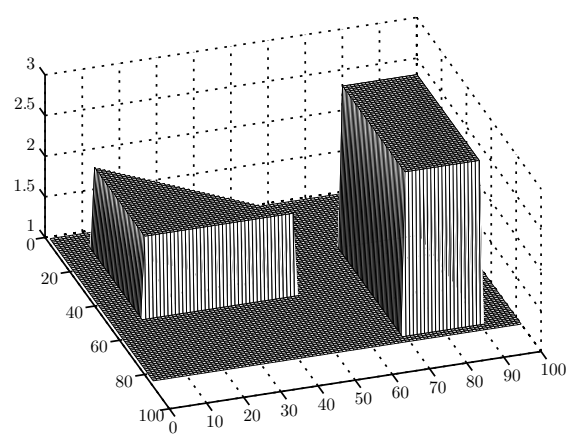

(c) Level set function $\phi=1 \wedge 2 \wedge 3$.

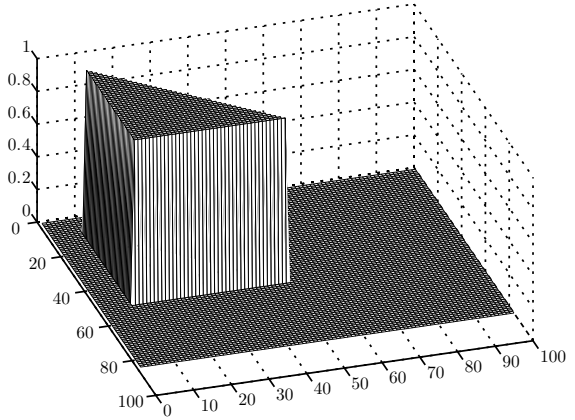

(e) Basis function $\psi_{2} \cdot \psi_{2}=0$ or 1 .

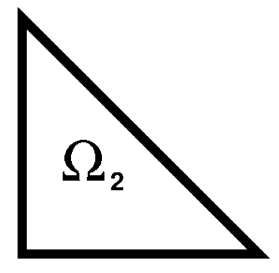

$\Omega_{1}$

(b) Partition $\Omega=\bigcup_{i=1}^{3} \Omega_{i}$.

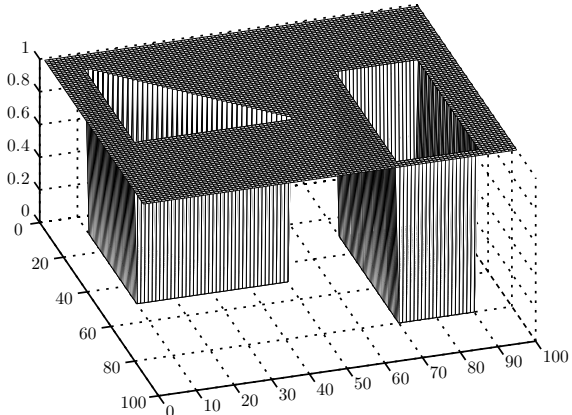

(d) Basis function $\psi_{1} \cdot \psi_{1}=0$ or 1 .

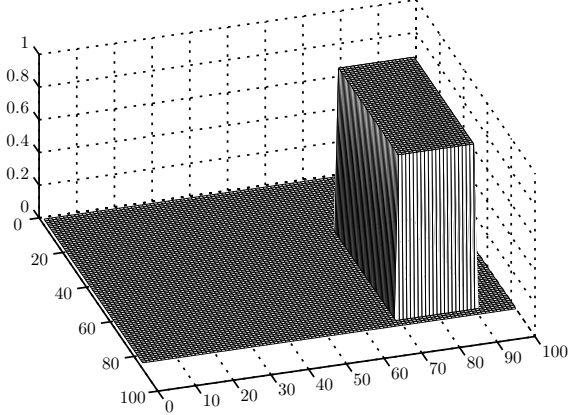

(f) Basis function $\psi_{3} \cdot \psi_{3}=0$ or 1 .

Figure 2. The relationship between an image function $u \in$ $B V(\Omega)$, the partition of $\Omega$ associated with the image, the piecewise constant level set function $\phi$ and the corresponding basis functions $\psi_{i}, i=1,2,3$. 
In Figure 2 we demonstrate the relationship between an image function $u$, the partition of $\Omega$ associated with this image, the piecewise constant level set function $\phi$ and the corresponding basis functions $\psi_{i}, i=1,2,3$. To ensure that equation (2.7) gives a unique representation of $u$, i.e., at convergence different values of $\phi$ should correspond to different function values $u(\phi)$ in (2.7), we introduce

$$
K(\phi)=(\phi-1)(\phi-2) \cdots(\phi-n)=\prod_{i=1}^{n}(\phi-i) .
$$

If a given function $\phi: \Omega \mapsto \mathbb{R}$ satisfies

$$
K(\phi)=0
$$

there exists a unique $i \in\{1,2, \ldots, n\}$ for every $x \in \Omega$ such that $\phi(x)=i$. Thus, each point $x \in \Omega$ can belong to one and only one phase if $K(\phi)=0$. Thus constraint (2.10) is used to guarantee that there is no vacuum and overlap between the different phases. In 27] some other constraints for the classical level set methods were used to avoid vacuum and overlap.

Based on the above observations, we propose to solve the following constrained minimization problem for segmenting an image $u_{0}$ :

$$
\min _{\substack{\mathbf{c}, \phi \\ K(\phi)=0}}\left\{F(\mathbf{c}, \phi)=\frac{1}{2} \int_{\Omega}\left|u-u_{0}\right|^{2} d x+\beta \sum_{i=1}^{n} \int_{\Omega}\left|\nabla \psi_{i}\right| d x\right\} .
$$

From (2.8), it is clear that the penalization term is just the sum of the length of the subdomain boundaries, and the penalization parameter $\beta>0$ controls the effect of these terms. Another approach could be to regularize $\phi$ directly in (2.11). This would make the algorithm simpler. Numerical experiments indicate that such an approach produces results that are nearly equally good. However, the total variation norm of $\phi$ is related to both the jumps and the length of the subdomain boundaries. Such a penalization will depend on the jumps, which is not preferable for some problems; see Figure 2(c).

If the image $u_{0}$ is a piecewise constant function and we take $\beta=0$, then any minimizer of (2.11) will give a function $u$ such that $u=u_{0}$, where $u$ is related to the minimizers $\mathbf{c}$ and $\phi$ as in (2.7).

\section{Minimization USing AUGmented LAgRAngian Method}

We use the augmented Lagrangian method to solve the constrained minimization problem (2.11). The augmented Lagrangian functional for this minimization problem is defined as

$$
L(\mathbf{c}, \phi, \lambda)=F(\mathbf{c}, \phi)+\int_{\Omega} \lambda K(\phi) d x+\frac{r}{2} \int_{\Omega}|K(\phi)|^{2} d x,
$$

where $\lambda \in L^{2}(\Omega)$ is the multiplier and $r>0$ is a penalty parameter. For the augmented Lagrangian method, it is not necessary to choose the penalization parameter $r$ very large. To find a minimizer for (2.11), we need to find the saddle points for $L$. We use the following Uzawa type algorithm to find a saddle point for $L(\mathbf{c}, \phi, \lambda)$. 
Algorithm 1. Choose initial values for $\phi^{0}$ and $\lambda^{0}$. For $k=1,2, \ldots$, do:

(1) Find $\mathbf{c}^{k}$ from

$$
L\left(\mathbf{c}^{k}, \phi^{k-1}, \lambda^{k-1}\right)=\min _{\mathbf{c}} L\left(\mathbf{c}, \phi^{k-1}, \lambda^{k-1}\right) .
$$

(2) Use (2.7) to update $u=\sum_{i=1}^{n} c_{i}^{k} \psi_{i}\left(\phi^{k-1}\right)$.

(3) Find $\phi^{k}$ from

$$
L\left(\mathbf{c}^{k}, \phi^{k}, \lambda^{k-1}\right)=\min _{\phi} L\left(\mathbf{c}^{k}, \phi, \lambda^{k-1}\right) .
$$

(4) Use (2.7) to update $u=\sum_{i=1}^{n} c_{i}^{k} \psi_{i}\left(\phi^{k}\right)$.

(5) Update the Lagrange-multiplier by

$$
\lambda^{k}=\lambda^{k-1}+r K\left(\phi^{k}\right)
$$

This algorithm has a linear convergence, and its convergence has been analyzed by Kunisch and Tai in [13] under a slightly different context. The algorithm has also been used by Chan and Tai in [5, 4] for elliptic inverse problems.

There are different methods to solve minimization problem (3.3). We have used the gradient method with a fixed step size to solve it. For a given old value of $\phi$, we get a new $\phi$ by

$$
\phi^{\text {new }}=\phi^{\text {old }}-\Delta t \frac{\partial L}{\partial \phi}\left(\mathbf{c}^{k}, \phi^{\text {old }}, \lambda^{k-1}\right) .
$$

The step size $\Delta t$ is chosen by a trial and error approach, and it is fixed during the whole iterative procedure. It is not necessary to solve the minimization problem (3.3) exactly. We terminate the gradient iteration (3.5) when

$$
\left\|\frac{\partial L}{\partial \phi}\left(\mathbf{c}^{k}, \phi^{\text {new }}, \lambda^{k-1}\right)\right\|_{L^{2}} \leq \frac{1}{10}\left\|\frac{\partial L}{\partial \phi}\left(\mathbf{c}^{k}, \phi^{k-1}, \lambda^{k-1}\right)\right\|_{L^{2}}
$$

is reached or else after each 400 iterations, whereupon we take $\phi^{k}=\phi^{\text {new }}$. To compute $\frac{d L}{d \phi}$ we utilize the chain rule to get

$$
\frac{\partial L}{\partial \phi}=\left(u-u_{0}\right) \frac{\partial u}{\partial \phi}-\beta \sum_{i=1}^{n} \nabla \cdot\left(\frac{\nabla \psi_{i}}{\left|\nabla \psi_{i}\right|}\right) \frac{\partial \psi_{i}}{\partial \phi}+\lambda \frac{\partial K}{\partial \phi}+r K \frac{\partial K}{\partial \phi} .
$$

It is easy to get $\partial u / \partial \phi, \partial \psi_{i} / \partial \phi$ and $\partial K / \partial \phi$ from (2.6), (2.7) and (2.9).

As $u$ is linear with respect to the $c_{i}$ values, we see that $L$ is quadratic with respect to $c_{i}$. Thus the minimization problem (3.2) can be solved exactly. Note that

$$
\frac{\partial L}{\partial c_{i}}=\int_{\Omega} \frac{\partial L}{\partial u} \frac{\partial u}{\partial c_{i}}=\int_{\Omega}\left(u-u_{0}\right) \psi_{i} d x, \quad \text { for } i=1,2, \ldots, n .
$$

Therefore, the minimizer of (3.2) satisfies an $n \times n$ linear system $A \mathbf{c}^{k}=\mathbf{b}$; there $A_{i j}=\left(\psi_{i}, \psi_{j}\right)_{L_{2}(\Omega)}$, and $b_{i}=\left(u_{0}, \psi_{i}\right)_{L_{2}(\Omega)}$.

$$
\sum_{j=1}^{n} \int_{\Omega}\left(\psi_{i} \psi_{j}\right) c_{i}^{k} d x=\int_{\Omega} u_{0} \psi_{i} d x, \quad \text { for } i=1,2, \ldots, n .
$$

In the above $\psi_{j}=\psi_{j}\left(\phi^{k}\right), \psi_{i}=\psi_{i}\left(\phi^{k}\right)$, and thus $\mathbf{c}^{k}=\left\{c_{i}^{k}\right\}_{i=1}^{n}$ depends on $\phi^{k}$. Since the number of phases, i.e., $n$, is typically small, we solve the equation $A \mathbf{c}^{k}=\mathbf{b}$ by an exact solver in our simulations. 
Remark 3.1. The updating for the constant values in (3.2) is ill-posed. A small perturbation of the $\phi$ function produces a large perturbation for the $\mathbf{c}$ values. Due to this reason, we have tried out a variant of Algorithm 1. In each iteration we alternate between (3.3) and (3.4), while (3.2) is only carried out if $\left\|K\left(\phi^{\text {new }}\right)\right\|_{L^{2}}<$ $\frac{1}{10}\left\|K\left(\phi^{\text {old }}\right)\right\|_{L^{2}}$. Here, $\phi^{\text {old }}$ denotes the value of $\phi$ when (3.2) was carried out the last time, and $\phi^{\text {new }}$ denotes the current value of $\phi$. If we use such a strategy, we can do just one or a few iterations for the gradient scheme (3.5) and Algorithm 1 is still convergent. This strategy is particularly efficient when the amount of noise is high.

Remark 3.2. In Algorithm 1, we give initial values for $\phi$ and $\lambda$. We first minimize with the constant values, and then minimize with the level set function. The multiplier is updated in the end of each iteration. In situations where good initial values for $\mathbf{c}$ are available, an alternative variant of Algorithm 1 may be used, i.e., we first minimize with the level set function followed by a minimization for the constant values and then update the multiplier.

\section{Strength AND WeAKness of OUR LEVEl SET FORMUlation}

In this section we will point out the main differences between a classical level set formulation and the variant we have proposed in this paper. First, while multiple level set methods [6, 25] utilize $n$ level set functions to represent $2^{n}$ distinct phases, our alternative level set formulation lets us represent the same number of phases using only one level set function. This gains in storage capacity.

Further, when minimizing (2.3), the associated Euler-Lagrange equations are [6. 25]

$$
\begin{aligned}
\frac{\partial \phi_{1}}{\partial t}=\delta\left(\phi_{1}\right)\{\nu & \operatorname{div}\left(\frac{\nabla \phi_{1}}{\left|\nabla \phi_{1}\right|}\right) \\
- & {\left[\left(\left(u_{0}-c_{1}\right)^{2}-\left(u_{0}-c_{3}\right)^{2}\right) H\left(\phi_{2}\right)\right.} \\
+ & \left.\left.\left(\left(u_{0}-c_{2}\right)^{2}-\left(u_{0}-c_{4}\right)^{2}\right)\left(1-H\left(\phi_{2}\right)\right)\right]\right\}, \\
\frac{\partial \phi_{2}}{\partial t}=\delta\left(\phi_{2}\right)\{\nu & \operatorname{div}\left(\frac{\nabla \phi_{2}}{\left|\nabla \phi_{2}\right|}\right) \\
- & {\left[\left(\left(u_{0}-c_{1}\right)^{2}-\left(u_{0}-c_{2}\right)^{2}\right) H\left(\phi_{1}\right)\right.} \\
+ & \left.\left.\left(\left(u_{0}-c_{3}\right)^{2}-\left(u_{0}-c_{4}\right)^{2}\right)\left(1-H\left(\phi_{1}\right)\right)\right]\right\},
\end{aligned}
$$

where both the Heaviside function $H(\phi)$ and the Delta function $\delta(\phi)$ are involved. Normally, these functions have to be regularized; see for example [3] for different approximations for $H_{\epsilon}(\phi)$ and $\delta_{\epsilon}(\phi)$. However, some numerical errors do occur when approximations are used for $H_{\epsilon}(\phi)$ and $\delta_{\epsilon}(\phi)$. For small $\epsilon$, the Delta function $\delta_{\epsilon}(\phi)$ is a function with sharp singular layers around the zero level curve of $\phi$. To achieve level set motion, a common approach is to replace $\delta_{\epsilon}(\phi)$ by $|\nabla \phi|$. But from numerical results we cannot conclude which of the schemes (i.e., $\delta_{\epsilon}(\phi)$ versus $\left.|\nabla \phi|\right)$ has the overall best performance. We avoid these problems in our formulation. The energy function for our approach is smooth and locally convex. The convergence for the Uzawa algorithm can be analyzed, and it has a linear convergence rate [13]. 
Also, $\phi$ is normally initialized as the signed distance function such as in (1.1). However, as the interface evolves, $\phi$ will generally drift away from its initialized value, i.e., the signed distance function. Moreover, $\phi$ may develop noisy features and steep gradients that are not amenable to finite-difference approximations. For this reason, it is advisable to reinitialize the level set function, i.e., force $\phi$ to satisfy the partial differential equation $|\nabla \phi|=1$. The reinitializing technique is superfluous in our formulation. Instead of reinitializing, we enforce $K(\phi)=0$ using a variational formulation. These strategies are akin.

One advantage in using classical level set methods is the ability to express geometrical quantities and motions. The length of the zero level curve $\Gamma$ and the area $A$ of the region inside $\Gamma$ are measured by $|\Gamma|=\int_{\Omega}|\nabla H(\phi)| d x$ and $|A|=\int_{\Omega}|H(\phi)| d x$. Further, the unit normal $\mathbf{N}$ and the mean curvature $\kappa$ to $\Gamma$ are defined by $\mathbf{N}=\frac{\nabla \phi}{|\nabla \phi|}$ and $\kappa=\nabla \cdot \mathbf{N}$. For our approach, there are some difficulties in finding a suitable way to represent the unit normal and the mean curvature for the curves.

Another shortcoming of our method is that higher order polynomials are used as basis functions to represent $u$ in (2.7). Further, the same basis functions are also used to find $\mathbf{c}$ in (3.9). To illustrate this weakness, we depict the linear, quadratic, cubic and quartic basis functions in Figure 3. In Figure 3 we see that one, and only

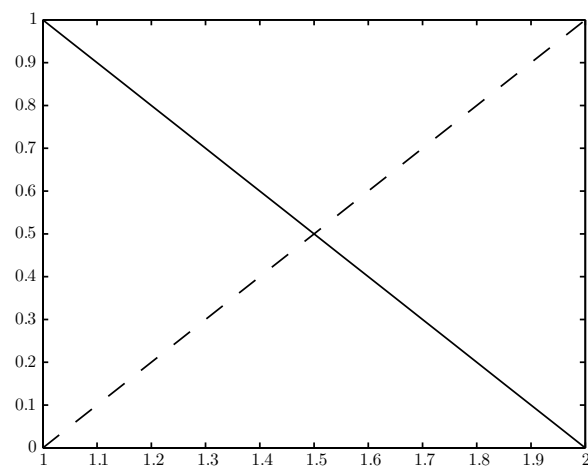

(a) Linear basis functions $\psi_{1}, i=1,2$.

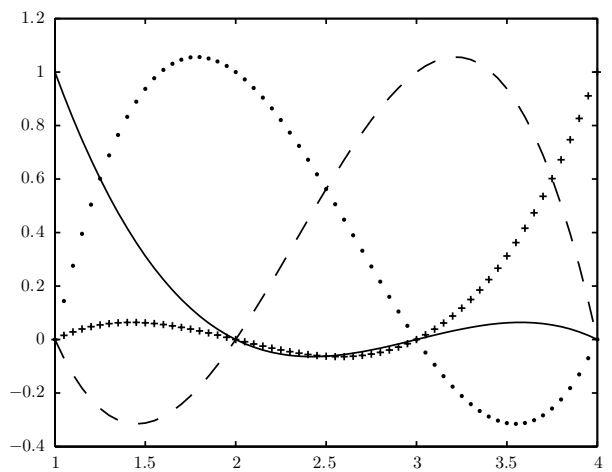

(c) Cubic basis functions $\psi_{1}$, $i=1,2, \ldots, 4$.

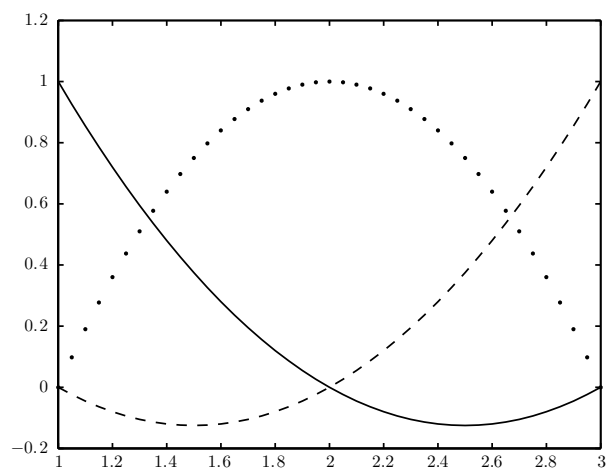

(b) Quadratic basis functions $\psi_{i}$.

$$
i=1,2,3 \text {. }
$$

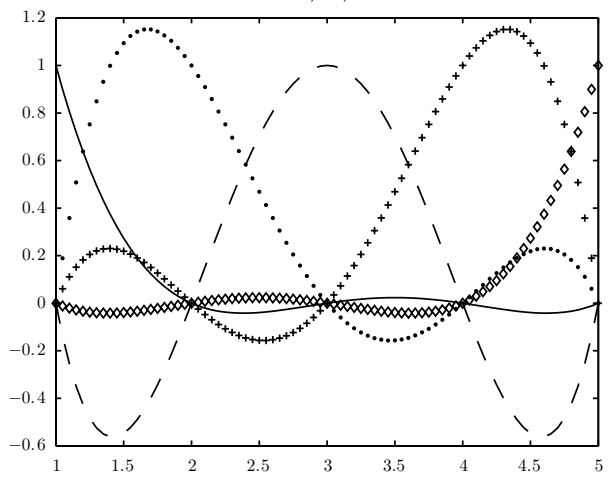

(d) Quartic basis functions $\psi_{i}$, $i=1,2, \ldots, 5$.

Figure 3. Different basis functions defined in (2.6). 
one, basis function is different from zero at each root when $K(\phi)=0$ is fulfilled. However, by inspecting the higher order basis functions $\psi_{i}$ in Figures 3(c) and (d), we see that even a small perturbation away from each root causes a significant change in the contribution from several of the basis functions. This sensitivity for perturbations makes the coefficient matrix in (3.9) ill-conditioned. To cure this problem we could search for other basis functions with better orthogonality properties, but that is outside the scope of this paper.

To this end, we emphasize that numerical tests have shown that proper initial guesses are needed using our approach. Fortunately we can use a simple strategy to approximate the constant coefficients $c_{i}$. Basically these constants are given by (3.9) if $\psi_{i}(\phi)$ is approximately known. Assume now that an observed signal $u_{0}$ should be segmented into $n$ phases. From the constraint $K(\phi)=0$ it follows that $\phi$ must take values in the set $\{1,2, \ldots, n\}$. As an approximation for $\phi$ we let $\phi=u_{0}$ and thereafter rescale $\phi$ such that $1 \leq \phi(x, y) \leq n$ for $(x, y) \in \Omega$. Using this approach we have a way to initialize $\phi$ and find $\psi_{i}(\phi)$ to get good initial guesses for the coefficients $c_{i}, i=1,2, \ldots, n$.

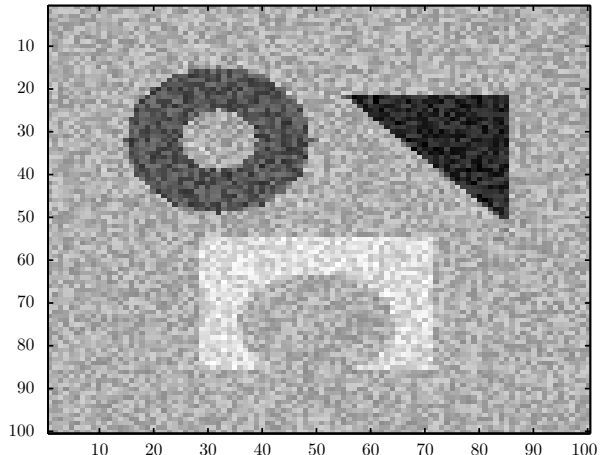

(a) Observed image $u_{o}(\mathrm{SNR} \approx 5.2)$.

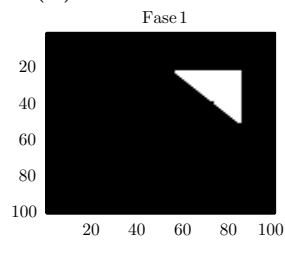

Fase 3

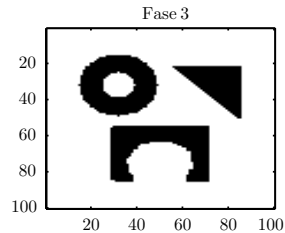

(c) Different phses using PCLSM.

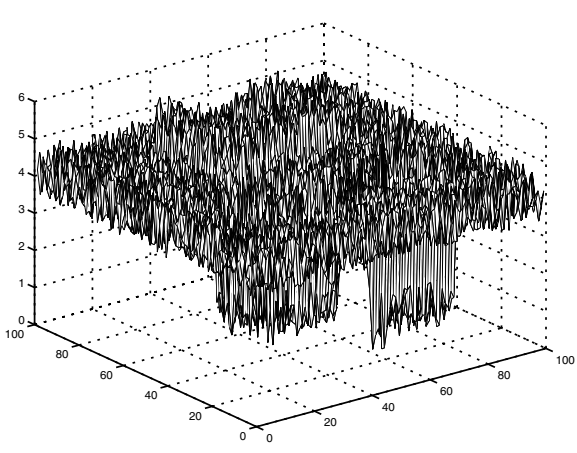

(b) Initial level set function $\phi$.

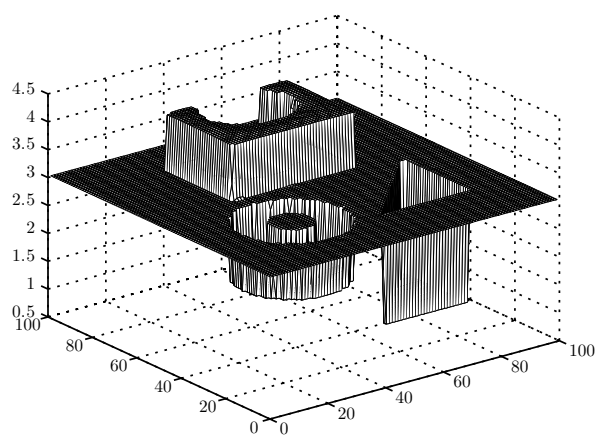

(d) At convergence $\phi$ approaches 4 constant values.

Figure 4. Each separate phase $\phi=1 \vee 2 \vee 3 \vee 4$ is depicted as a bright region in Figure \(c). 


\section{Numerical Results}

In this section we validate the piecewise constant level set method (PCLSM) with numerical examples. We only consider two-dimensional cases and restrict ourselves to gray-scale images, but the model can handle any dimension and can be extended to vector-valued images as well. Both synthesized images, natural scenes and an MR image are evaluated in this section. Our results will be compared with other related works [6, 25, 23. The original image is known for some cases we evaluate here. Thereupon it is trivial to find the perfect segmentation result. To complicate such a segmentation process we typically expose the original image with Gaussian distributed noise and use the polluted image as the observation data $u_{0}$. To indicate the amount of noise that appears in the observation data, we report the signal-to-noise-ratio:

$$
\mathrm{SNR}=\frac{\text { variance of data }}{\text { variance of noise }}
$$

To demonstrate a 4-phase segmentation, we begin with a noisy synthetic image containing 3 objects (and a background) as shown in Figure 4(a). This is the same image that Chan and Vese used to examine their multiphase algorithm [6, 25]. A careful evaluation of our algorithm is reported in Figure 4. The observation data $u_{0}$

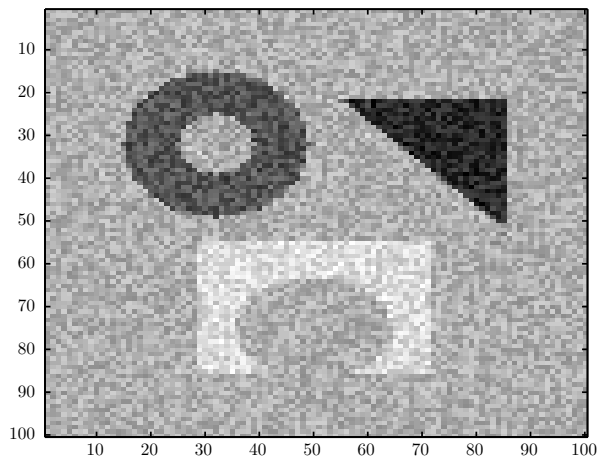

(a) Observed image $u_{o}(\mathrm{SNR} \approx 5.2)$.
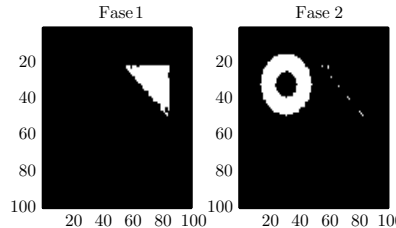

4

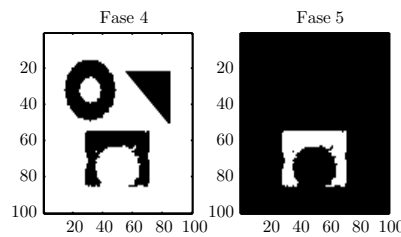

(c) Different phases using PCLSM.

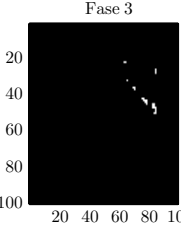

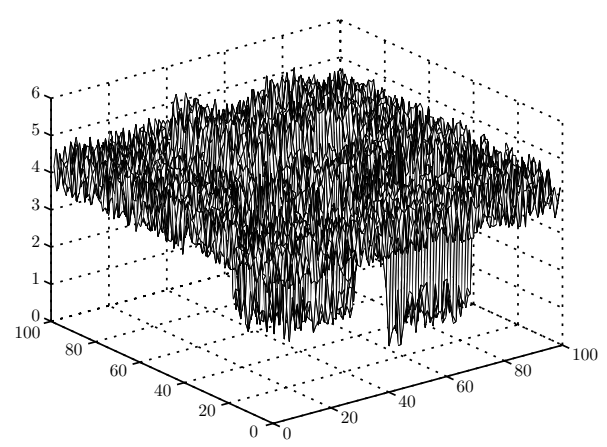

(b) Initial level set function $\phi$.

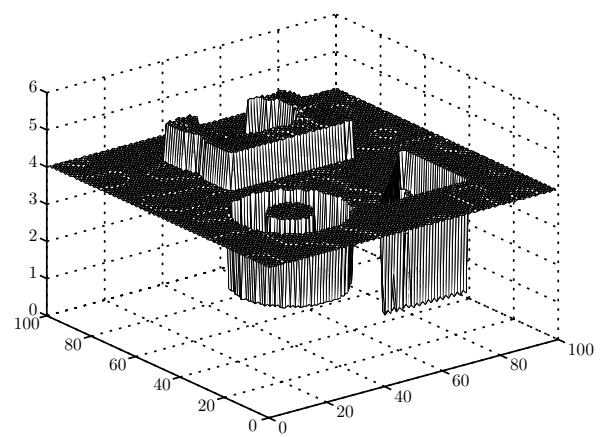

(d) At convergence $\phi$ approaches 4 constant values.

FiguRE 5. Each separate phase $\phi=1 \vee 2 \vee 3 \vee 4 \vee 5$ are depicted as a bright region in Figure 5 (c). 
is given in Figure 4(a), and the only assumption we make is that a 4-phase model should be utilized to find the segments. In Figure 4(d) the $\phi$ function is depicted at convergence (less than 1000 iterations). $\phi$ approaches the predetermined constants $\phi=1 \vee 2 \vee 3 \vee 4$. Each of these constants represents one unique phase as seen in Figure 4(c). Our result is in accordance with what Chan and Vese reported in $[6,25]$.

In many applications the number of objects to detect is not known a priori. A robust and reliable algorithm should find the correct segmentation even when the exact number of phases is not known. By introducing a model with more phases than one actually needs, we can find the correct segmentation if all superfluous phases are empty when the algorithm has converged. To see if our algorithm can handle such a case we use Figure 5(a) as the observation image and utilize a 5-phase model. The results are reported in Figure 5 .

One of the 5 phases must be empty if a 5-phase model is used to find a 4-phase segmentation. Due to the high noise level some pixels can easily be misclassified and contribute to the phase that should be empty. The level set function shown in Figure 5(d) approaches the constants $\phi=1 \vee 2 \vee 4 \vee 5$, except from the few misclassified pixels where $\phi=3$ as seen in Figure 5(c). By comparing Figure 4(c) (where a 4-phase model is used) and Figure 5(c) (where a 5-phase model is used),

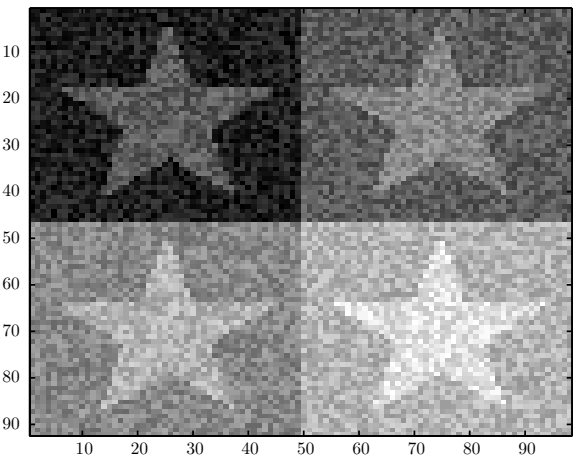

(a) Observed image $u_{o}(\mathrm{SNR} \approx 10.6)$.
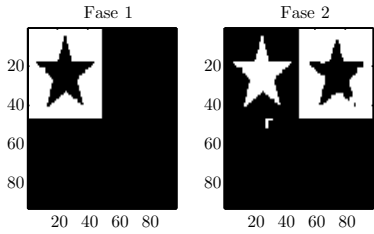

$\begin{array}{llll}20 & 40 & 60 & 80\end{array}$
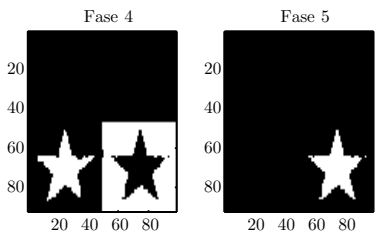

(c) Different phases using PCLSM.

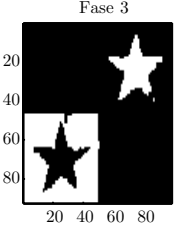

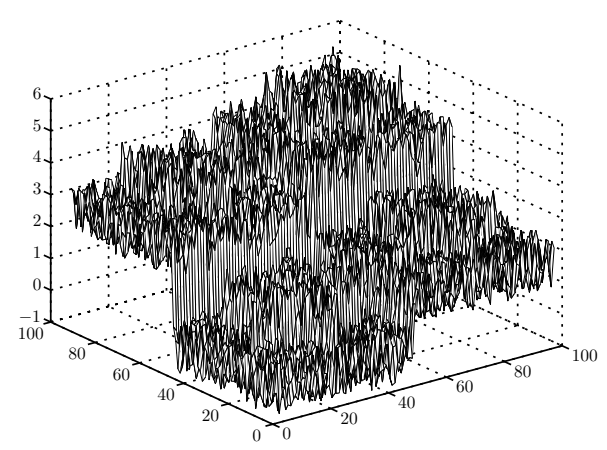

(b) Initial level set function $\phi$.

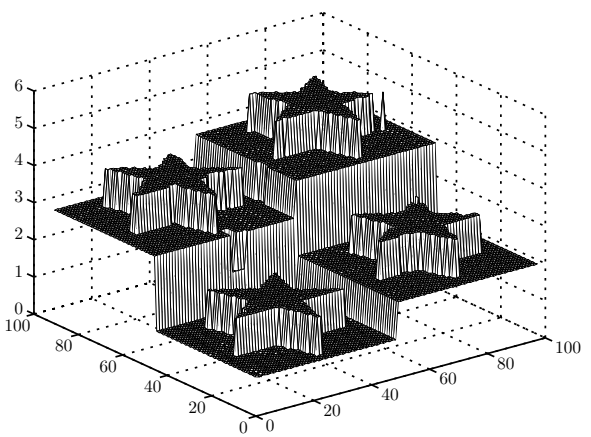

(d) At convergence $\phi$ approaches 4 constant values.

Figure 6. Each separate phase $\phi=1 \vee 2 \vee 3 \vee 4 \vee 5$ is depicted as a bright region in Figure 6(c). 
we observe only small changes in the segmented phases, except from the extra nonempty phase $\phi=3$ in Figure 5(c). For a test like this, we cannot control which phase that will end up empty.

In the example below we use PCLSM to find a 5-phase segmentation. We use a noisy synthetic image containing 4 stars on 4 different backgrounds, as shown in Figure 6(a). There are only 5 different phases in this image. The star in the upper left corner belongs to the same phase as the background in the upper right corner. Further, the star in the upper right corner belongs to the same phase as the background in the lower left corner, and the star in this corner belongs to the same phase as the background in the lower right corner. In total that gives us 5 different phases. Almost no misclassification was observed in this test. By using one level set function, we manage to find all 5 objects, as seen from Figure 6(c). This is in contrast to classical level set methods, where 3 level set functions must be utilized to get the same result.

If an image actually consists of 5 objects, but a 4-phase model is utilized in the segmentation procedure, one may hope that 2 of the 5 objects merge into 1 new object without affecting the segmentation result for the other objects. Below we depict our result using Figure 6(a) as the observation data in a 4-phase segmentation. By comparing Figures 5(c) and 7(c) we see that phase $\phi=2$ and phase $\phi=3$

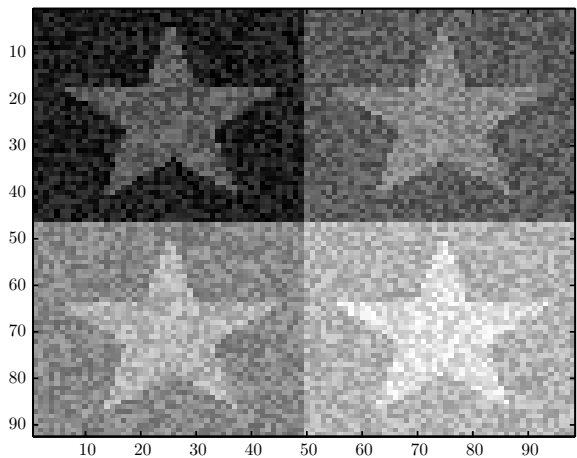

(a) Observed image $u_{o}(\mathrm{SNR} \approx 10.6)$.
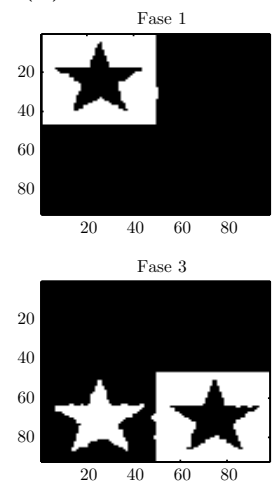

(c) Different phases using PCLSM.

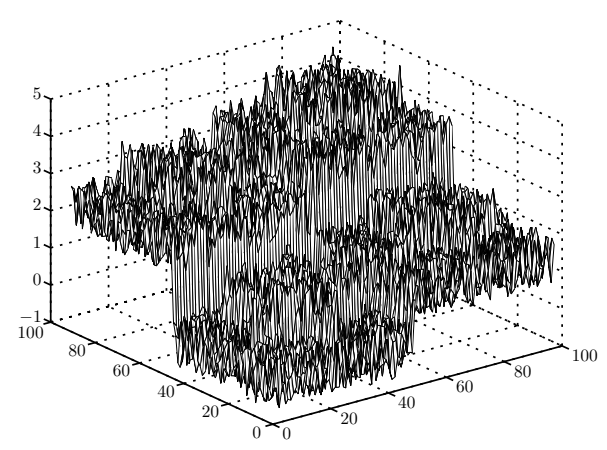

(b) Initial level set function $\phi$.

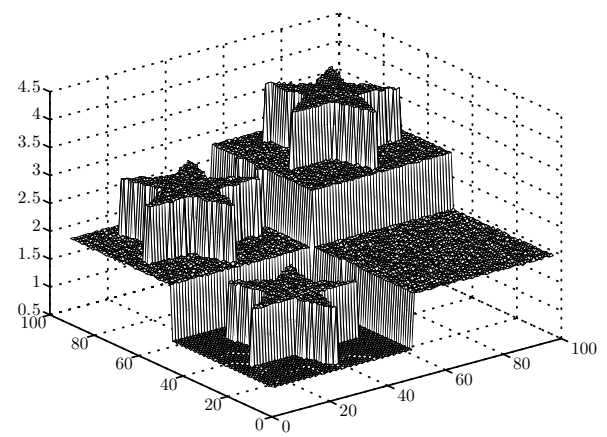

(d) At convergence $\phi$ approaches 4 constant values.

Figure 7. Each separate phase $\phi=1 \vee 2 \vee 3 \vee 4$ is depicted as a bright region in Figure 6(c). 


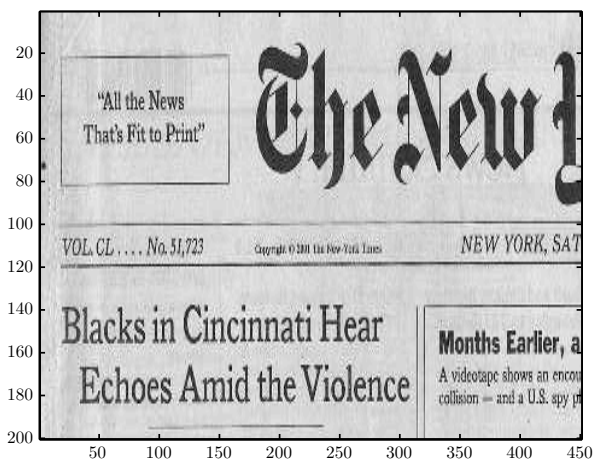

(a) Old newspaper.

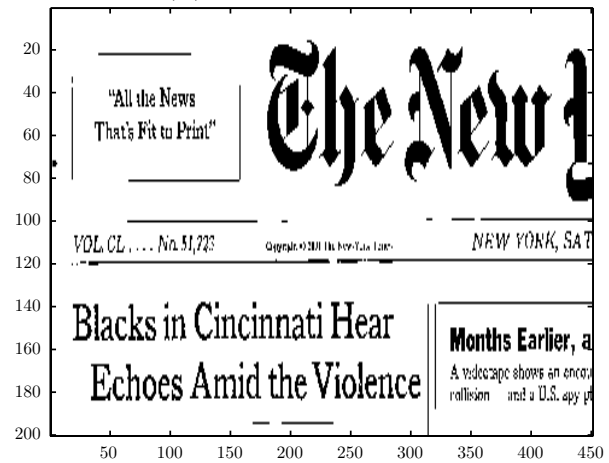

(c) Result using PCLSM.

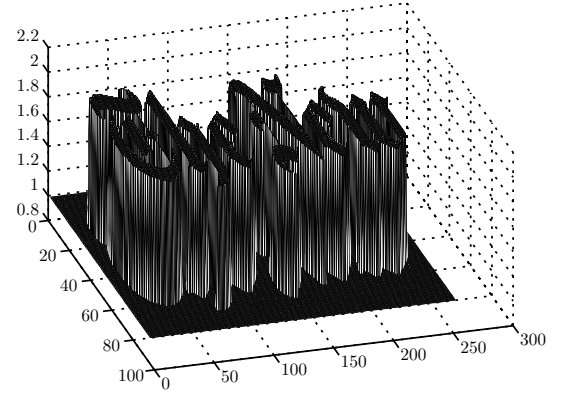

(b) A small portion of $\phi$.

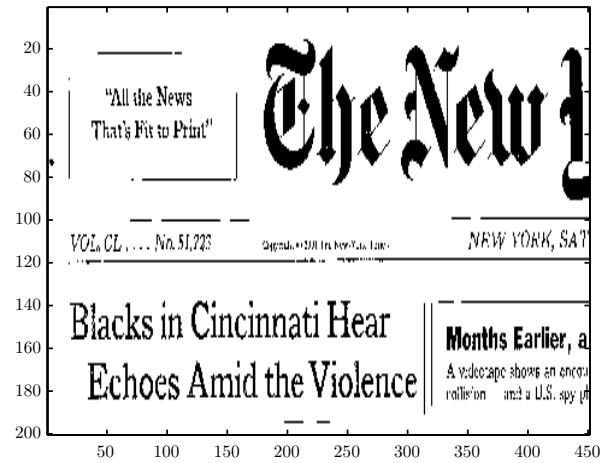

(d) Result using CVM.

FIGURE 8. For the purpose of illustration only a small partition of the level set function $\phi$ is depicted in the upper right corner. We see that $\phi$ takes two constant values, as it should.

in Figure 5(c) have merged into one phase $\phi=2$ in Figure 7(c). All other phases seem to be unaffected by this.

All the examples above indicate that our algorithm is an interesting alternative to the multiphase algorithm [6, 25, where the standard level set formulation is utilized. We have shown that our algorithm is robust with respect to noise. As in 6, 25] we have shown that the exact number of phases does not have to be known a priori. Redundant phases can disappear in the sense that they will end up empty. Below we proceed with some examples using real images (some natural scene and one MR image). First we demonstrate that PCLSM can be used to extract characters or numbers from images. We begin with an image of an old newspaper where only two phases are needed; one phase to represent the characters and one phase to represent the remaining. To evaluate the segmentation process, the Chan/Vese method [6, 25], for short (CVM), is examined using the same input image. The results achieved with the segmentation techniques PCLSM and CVM are shown in Figures $8(\mathrm{c})$ and $8(\mathrm{~d})$, respectively. Few differences between the segmented images were observed, but a careful inspection of all characters in Figures 8(c) and 8(d) reveals that PCLSM preserves small details somewhat better than CVM. In this paper we will mainly focus on the segmentation process, but let us briefly describe a way to identify the characters separated by phase $\phi=2$ given in 
Figure 8(b). First one can isolate each character in this phase by a peak-and-valley method [21]. Thereafter, to identify the isolated characters, the center of mass for each character is aligned to the center of mass for all character templates in a database. Thereafter each isolated character is registered to all templates. Finally the difference between each registered character and all templates are measured. The template corresponding to the smallest difference in $L_{2}$-norm is chosen to be the solution.

In the next example we illustrate a 2-phase segmentation on a real car plate image. Locating and reading car plates is a well-known problem, and there is a number of commercial software available. Below we demonstrate that our level set method can be used in such an application. Since character and number recognitions are the main target in this example, we assume the location of the car plate to be known.

A perfect segmentation was found with both PCLSM and CVM if Figure 9(a) was used as the observation data. We challenge these two segmentation techniques by adding Gaussian distributed noise to the real image and use the polluted image in Figure 9(b) as the observation data. With this amount of noise both PCLSM and CVM miss some details along the edges for the characters and numbers. By increasing the regularization term for CVM we obtain smoother edges but then each character or number may be broken into several pieces. From Figure 9(c) we see that each character and number appears as unbroken.

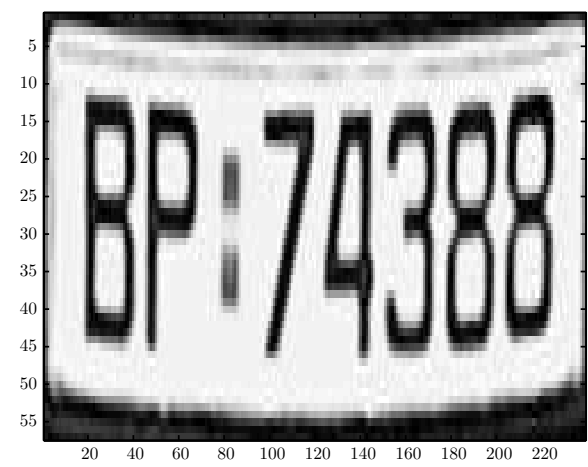

(a) Real image of a car plate.

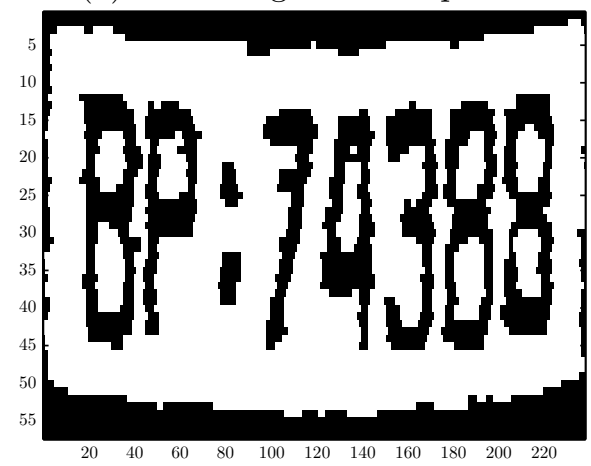

(c) Segmented with PCLSM.

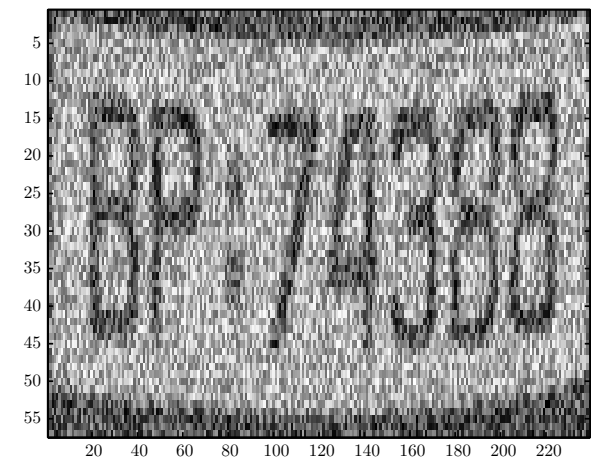

(b) Initial image $(\mathrm{SNR} \approx 1.7)$.

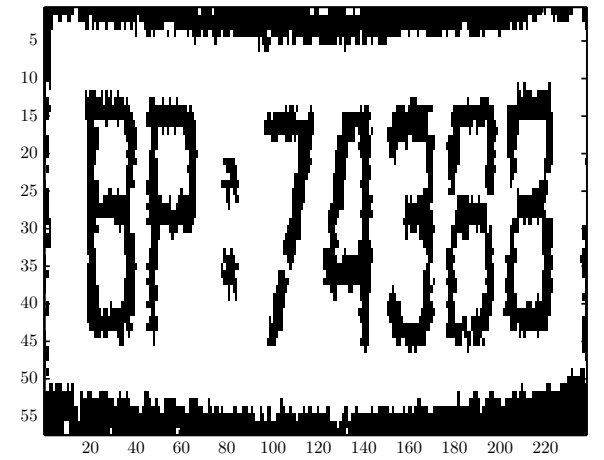

(d) Segmented with CVM.

Figure 9. Character and number segmentation from a car plate. 


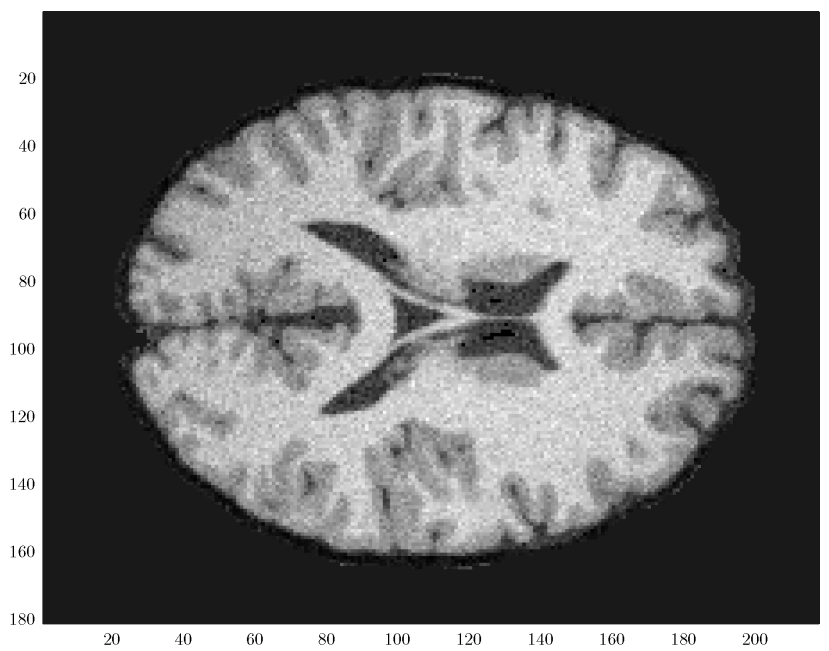

FIGURE 10. MRI image with a change in the intensity values going from left to right caused by the nonuniform RF-puls.

The number of iterations needed for 2-phase problems were almost equal for our scheme and related schemes [6, 25]. When the number of phases becomes large, $\Delta t$ in (3.5) must be chosen small. This may lead to an increase in the number of iterations for some examples, compared with [6, 25].

In our next example segmentation of an MR image is demonstrated. The image in Figure 10 is available to the public at http://www.bic.mni.mcgill.ca/brainweb/ . These realistic MRI data are used by the neuroimaging community to evaluate the performance of various image analysis methods in a setting where the truth is known. For the image used in this test the noise level is $7 \%$ and the nonuniformity intensity level of the RF-puls is $20 \%$; see http://www.bic.mni.mcgill.ca/brainweb/ for details concerning the noise level percent and the intensity level of the RF-puls. Both PCLSM and SPM 23 are used to segment the above MRI phantom, and the results are depicted in Figure 11. SPM is a statistical segmentation software program for processing MRI data for research and clinical use. In Figure 11 there are three tissue classes that should be identified; phase 1: cerebrospinal fluid, phase 2: gray matter, phase 3: white matter. Because of this, four phases were used, but we do not depict the background phase here. The main difference between PCLSM and SPM is their ability to handle noisy data. SPM is adjusted for MR data, but each phase obtained with SPM is perforated with misclassified pixels. The same problem does not occur with PCLSM.

As a final example we consider a scattered data problem. Here the goal is to connect single observation points and form continuous objects. The lights in a satellite image of Europe by night act as scattered data points. The intensity from each light source is the same, but the density of data points fluctuate within the satellite image. Generally it is hard to find features by connecting single observation points. Models based on edge stopping functions will fail completely for applications like this. We do not reckon our model as well suited for scattered data problems, but by following the proposition from Remark 3.1 in Section 3 we managed to get the result in Figure 12(c). This result is comparable with what Chan and Vese 
report in [3] for the same satellite image. In Figures [12(b)-(d) we demonstrate the effect of the total variation norm for $\psi_{i}$ in (2.11). Weighting these terms too softly causes an image with perforated regions as seen in Figure 12(b). On the other hand, if these terms are weighted too hard all sparse regions disappear, as seen in Figure $12(\mathrm{~d})$.

As can be seen, our model is easy to extend to higher-dimensional problems. In fact, some experiments have already been carried out for three-dimensional problems, and the results seem as good as for two-dimensional problems. In our future work, it would be valuable to search for basis functions with better orthogonality properties. An interesting approach could be to investigate if Legendre, Chebyshev or Hermite polynomials make the coefficient matrix (3.9) less ill conditioned. As our method is variational, the minimization problems can be solved by fast methods. At the moment, we are working on a Newton type of method to accelerate the convergence.

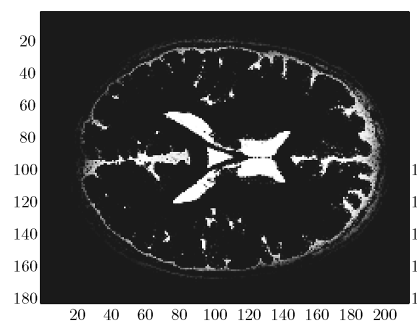

(a) SPM: phase 1 .

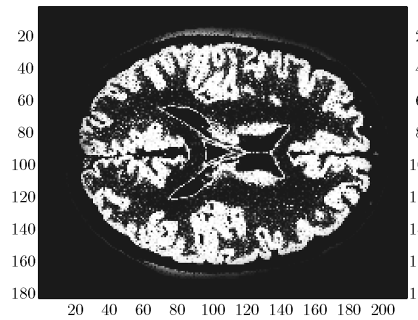

(d) SPM: phase 2 .

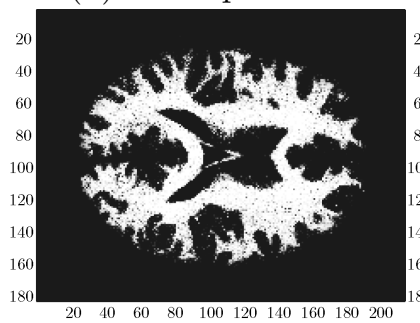

(g) SPM: phase 3 .

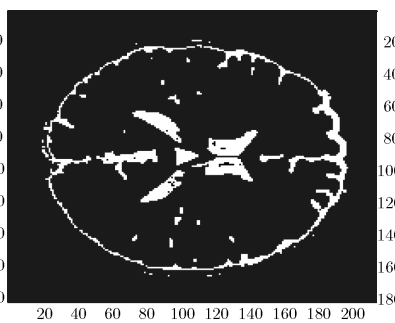

(b) PCLSM: phase 1 .

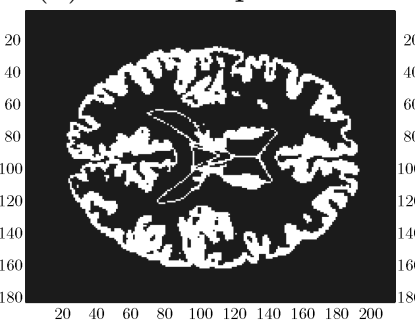

(e) PCLSM: phase 2 .

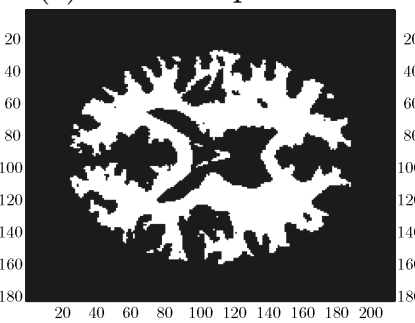

(h) PCLSM: phase 3.

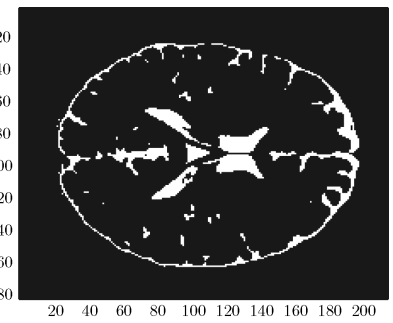

(c) Exact: phase 1 .

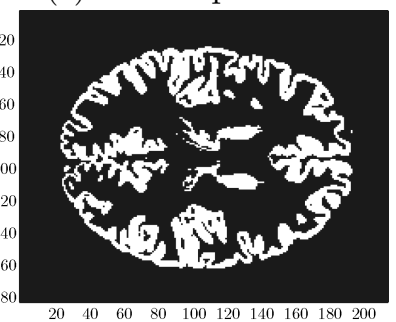

(f) Exact: phase 2 .

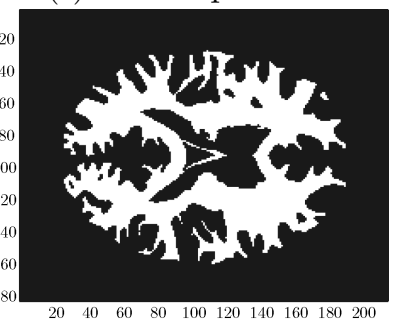

(i) Exact: phase 3 .

Figure 11. SPM is adjusted for MR data, but it seems hard for SPM to handle noise properly. All phases found by PCLSM match the exact phases in a better way than SPM does. 


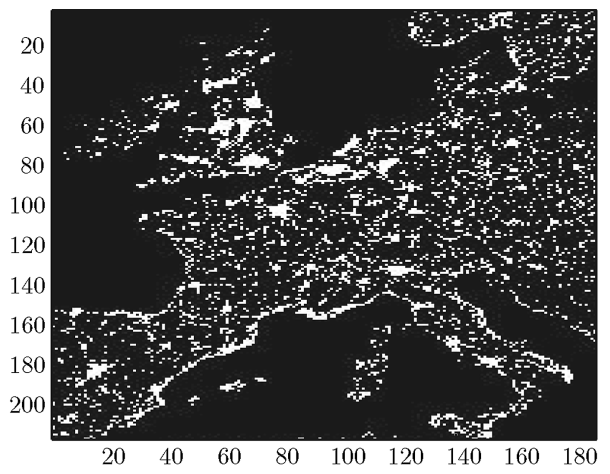

(a) Scattered data points.

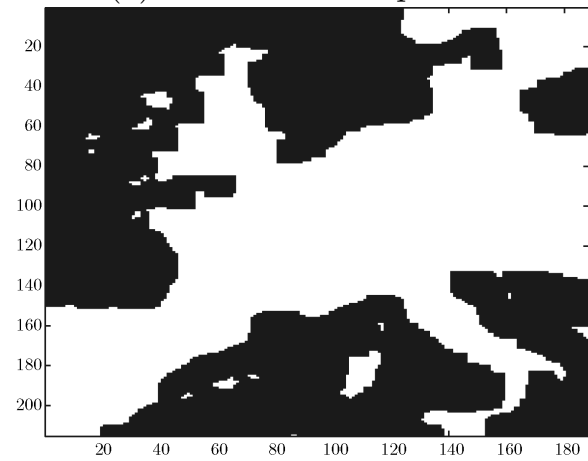

(c) Regularization $\beta=7$.

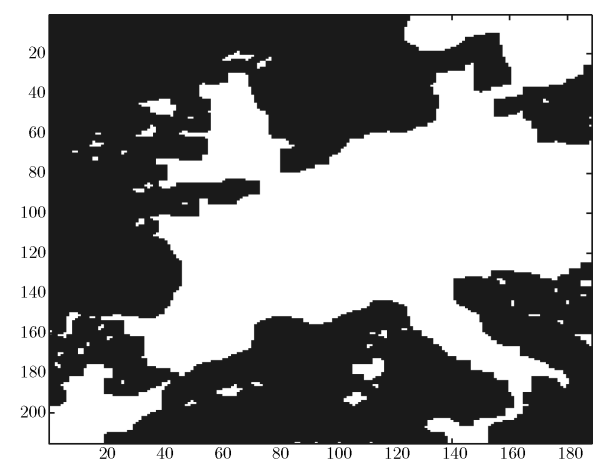

(b) Regularization $\beta=6$.

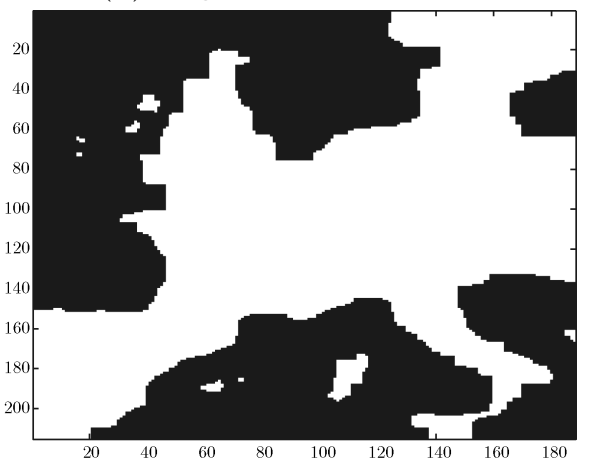

(d) Regularization $\beta=9$.

FIGURE 12. Scattered data are connected to form an object.

\section{ACKNOWLEDGMENT}

The authors thank Stanley Osher for valuable comments on a draft of this paper and for bringing our attention to references [16, 7].

\section{REFERENCES}

1. M. Burger, A framework for the construction of level set methods for shape optimization and reconstruction, Interfaces Free Bound, 5 (2003), pp. 301-329. MR1998617 (2004f:49077)

2. M. Burger, B. HACKL, AND W. RING, Incorporating topological derivatives into level set methods, J. Comput. Phys, 194 (2004), pp. 344-362. MR2033389(2004j:49062)

3. T. Chan And L. A. Vese, Active contours without edges, IEEE Image Proc., 10 (2001), pp. 266-277.

4. T. F. Chan AND X.-C. TAI, Identification of discontinuous coefficients in elliptic problems using total variation regularization, SIAM J. Sci. Comput., 25 (2003), pp. 881-904. MR2046116 (2005c:65091)

5. T. F. ChAN AND X.-C. TAI, Level set and total variation regularization for elliptic inverse problems with discontinuous coefficients, J. Comput. Physics, 193 (2003), pp. 40-66. MR2022688 (2004j:65170)

6. T. F. ChAN AND L. A. VeSE, Image segmentation using level sets and the piecewise constant Mumford-Shah model, Tech. Rep. CAM00-14, UCLA Dep. Math.

7. S. Chen, B. Merriman, M. Kang, R. E. Cafilsch, C. Ratsch, L.-T. Cheng, M. Gyure, R. P. Fedkiw, And S. Osher, Level set method for thin film epitaxial growth, Tech. Rep. CAM00-03, UCLA Math. Dep., 2000. 
8. S. Esedoglu And Y.-H. R. Tsai, Threshold dynamics for the piecewise constant MumfordShah functional, Tech. Rep. CAM04-63, UCLA Dep. Math, 2004.

9. L. C. Evens And R. F. Gariepy, Measure therory and fine properties of functions, 1992.

10. F. Gibou And R. FedKiw, A fast hybrid k-means level set algorithm for segmentation, tech. rep., Stanford, 2002 (in review).

11. E. Hodneland, Segmentation of digital images, cand. scient thesis, Dep. Math., University of Bergen, 2003. Available online at http://www.mi.uib.no/ tai/.

12. M. Kass, A. Witkin, And D. Terzopoulos, Snakes, active contour models, Int. J. of Comp. Vision, 1 (1988), pp. 321-331.

13. K. Kunisch AND X.-C. TAI, Sequential and parallel splitting methods for bilinear control problems in Hilbert spaces, SIAM J. Numer. Anal., 34 (1997), pp. 91-118. MR11445731|(98b:49025)

14. J. Lie, M. LySAKER, AND X.-C. TAI, A binary level set model and some applications to image processing, Tech. Rep. CAM04-31, UCLA Dep. Math., 2004.

15. B. Merriman, J. K. Bence, and S. J. Osher, Motion of multiple functions: a level set approach, J. Comput. Phys., 112 (1994), pp. 334-363. MR.1277282 (95a:65169)

16. B. Merriman, R. Caflisch, S. Osher, C. Ratsch, S. Chen, M. Kang, and M. Gyure, Island dynamics and level set methods for continuum modeling of epitaxial growth, in Applied and industrial mathematics, Venice-2, 1998, Kluwer Acad. Publ., Dordrecht, 2000, pp. 145171. MR. 1755326

17. D. Mumford AND J. Shah, Optimal approximation by piecewise smooth functions and associated variational problems, Comm. Pure Appl. Math., 42 (1989), pp. 577-685. MR0997568 (90g:49033)

18. S. Osher And J. A. Sethian, Fronts propagating with curvature dependent speed: Algorithms based on Hamilton-Jacobi formulations, J. Comput. Phys., 79 (1988), pp. 12-49. MR0965860 (89h:80012)

19. C. Samson, L. Blanc-Féraud, G. Aubert, and J. Zerubia, A level set model for image classification, IJCV, 40 (2000), pp. 187-197.

20. _ A variational model for image classification and restoration, TPAMI, 22 (2000), pp. $460-472$.

21. C. J. Setchell, Applications of computer vision to road-traffic monitoring, Ph.D. thesis, Department of Computer Science, University of Bristol, 1997. Available online at http://www.cs.bris.ac.uk/Tools/Reports/Abstracts/1997-setchell.html.

22. B. Song And T. Chan, A fast algorithm for level set based optimization, Tech. Rep. CAM0268, UCLA Dept. Math., 2002.

23. SPM, http://afni.nimh.nih.gov/afni/.

24. M. Sussman, P. Smereka, And S. Osher, A level set approach for computing solutions to incompressible two phase flow, J. Comput. Phys, 114 (1994), pp. 146-159.

25. L. A. VeSE AND T. F. ChAn, A multiphase level set framework for image segmentation using the Mumford and Shah model, International Journal of Computer Vision, 50 (2002), pp. 271293.

26. J. Weickert and G. Kühne, Fast methods for implicit active contour models, in Geometric level set methods in imaging, vision, and graphics, Springer, New York, 2003, pp. 43-57. MR 2070064

27. H.-K. ZhaO, T. Chan, B. Merriman, and S. Osher, A variational level set approach to multiphase motion, J. Comput. Phys., 127 (1996), pp. 179-195. MR1408069 (97g:80013)

Department of Mathematics, University of Bergen, Bergen, Norway

E-mail address: johanl@mi.uib.no

Department of Mathematics, University of Bergen, Bergen, Norway

Current address: Simula Research Lab, Norway

E-mail address: mariul@simula.no

Department of Mathematics, University of Bergen, Bergen, Norway

E-mail address: tai@mi.uib.no 